\title{
The Nappi-Witten string in the light-cone gauge ${ }^{\dagger}$
}

\author{
P. Forgács \\ Research Institute for Particle and Nuclear Physics \\ H-1525 Budapest 114, P.O.B. 49, Hungary \\ P.A. Horváthy \\ Département de Mathématiques \\ Université de Tours \\ Parc de Grandmont, F-37200 TOURS, France \\ Z. Horváth and L. Palla \\ Institute for Theoretical Physics \\ Roland Eötvös University \\ H-1088 Budapest, Puskin u. 5-7, Hungary
}

\begin{abstract}
Some of the motivations for as well as the main points of the quantization of the Nappi Witten string in the light cone gauge are reviewed.
\end{abstract}

\footnotetext{
$\dagger$ to appear in the E.P. Wigner memorial volume of Acta Physica Hungarica
} 
Recently the problem of strings propagating in various classical field backgrounds has received an increased interest [1-5]. The Nappi Witten string is a particular example of this type: it describes the propagation of a bosonic string in a particular gravitational wave and axion field background. These classical fields were obtained in [3] from an ungauged WZW model based on $E_{2}^{c}$, the central extension of the two dimensional Euclidean group. In [3] it was also shown, that these background fields solve the one loop $\beta$-function equations.

In [4] the highest weight representations of the Kac-Moody (KM) algebra corresponding to this WZW model were studied and it was pointed out that there are three types of Kac-Moody representations with unitary base. These three possibilities correspond to the three different unitary representations of the zero grade algebra (ZGA) (which is nothing but the Lie algebra of $E_{2}^{c}$ ). These three representations are distinguished by the number of ZGA highest/lowest weight vectors they contain and by the eigenvalue, $t$, of the central element. Type I representations contain neither ZGA highest nor lowest weight vectors and are characterized by $t=0$. Type II representations contain a ZGA lowest weight state and are characterized by $t>0$, while in Type III representations there is a ZGA highest weight state and all the states have $t<0$.

The spectrum and some scattering processes of the string theory obtained by adding 22 flat Euclidean coordinates to the $E_{2}^{c}$ WZW model have been investigated in [5]. Here we analyze this string theory, that we call Nappi Witten string, from a different point of view. The problem is that all the KM representations based on Type I-III ZGA representations are plagued by the presence of negative norm states. These negative norm states then appear in the string state space and one may ask whether the Virasoro conditions are enough to remove them from the physical subspace. There are at least two examples where the Virasoso conditions do indeed guarantee - albeit in a slightly different way - that the physical subspace contains only states with non-negative norms: the ordinary bosonic string propagating on a flat $D=26$ dimensional Minkowski space [6] and the example of the $S U(1,1)$ string [7]. The difference between these two cases lies in the fact that for the $S U(1,1)$ string one has to truncate the string state space to contain only a restricted set of $\widehat{S U}(1,1)$ representations [7], while for the bosonic string on Minkowski space no such truncation is necessary.

Recently, following the method outlined in [8] for the bosonic string on Minkowski space, we proved an analogous theorem [9] for the Nappi Witten string as defined above. This theorem states, that the Virasoro conditions are indeed enough to guarantee that the states in the physical subspace have non negative norms at least when we use KM representations having Type II states at the base and $1-t /(2 k)>0$ or when we use KM representations with Type III base and $1+t /(2 k)>0$. (Here $k$ denotes the parameter of the WZW model, that appears also as the level of the KM algebra). 
Although the proof of our theorem makes it possible to determine the global $E_{2}^{c} \times E_{2}^{c}$ quantum numbers of the physical states it offers very little insight into the structure of the physical subspace. Therefore, in the present paper, we reconsider the quantization of the Nappi Witten string in the light-cone gauge using the old fashioned operator approach. The motivation to consider the light-cone quantization comes from various considerations:

- This way one works with positive norm states only, thus the spectra one finds are the physical ones.

- One can confirm the spectra obtained by covariant methods and compare the two methods on an example other than that of a string propagating on flat Minkowski space.

- The light-cone quantization may give new insight into the interpretation of the various peculiar states of the Nappi-Witten string.

- One may obtain a physical interpretation of the restriction on the allowed $\widehat{E}_{2}^{c}$ representations (i.e. the $t$ range) found in our theorem.

- The direct determination of the physical states makes the analysis of modular invariance readily accessible.

The viability of light cone quantization in this case is based on an observation of Horowitz and Steif [2]: the light-cone gauge can be imposed in a curved spacetime only if it admits a covariantly constant null vector; i.e. if it describes a plane fronted gravitational wave (an axion field may also be present).

The paper is organized as follows: in chapter 1. we describe the action and the classical Hamiltonian in the light-cone gauge. Chapter 2. contains the discussion of the global symmetries. The mode expansion and quantization is carried out in chapter 3, while the mass operator, the energy spectrum and the restrictions on the physical states are analized in chapter 4 . In chapter 5 . we discuss the problems related to the determination of the critical dimension and describe the details of the chiral algebra. Chapter 6 . is devoted to a brief study of the consequences of dropping the previous limitation on the total momentum of the center of mass. Finally, in chapter 7. the one loop partition function is derived and its modular properties are analyzed. We make our conclusions in chapter 8 .

1. The action and Hamiltonian in the light-cone gauge

As a preparation we transform the spacetime metric and antisymmetric tensor field of the Nappi-Witten solution

$$
d s^{2}=\left(d a^{i}\right)^{2}-2 d U\left(d V+\frac{1}{2} \epsilon_{i j} a^{i} d a^{j}\right)+b d U^{2} ; \quad b_{i j}=U \epsilon_{i j}
$$

$(i, j=1,2)$ into a form for which the vanishing of the anomaly has been shown at all loop orders, and which is also more convenient for the light-cone quantization. Indeed making the gauge transformation $b_{\mu \nu} \rightarrow b_{\mu \nu}+2 \partial_{[\mu} \lambda_{\nu]}(\mu=U, V, i)$ with 
$\lambda_{i}=\frac{1}{2} U \epsilon_{i j} a^{j}$ on the antisymmetric tensor field and rotating the $a^{i}$ fields by an angle $U / 2$ :

$$
\begin{aligned}
& x^{1}=a^{1} \cos \frac{U}{2}+a^{2} \sin \frac{U}{2} \\
& x^{2}=-a^{1} \sin \frac{U}{2}+a^{2} \cos \frac{U}{2}
\end{aligned}
$$

we get

$$
d s^{2}=\left(d x^{i}\right)^{2}-2 d U d V+\left(b-\left(x^{i}\right)^{2} / 4\right) d U^{2} ; \quad b_{i u}=-(1 / 2) \epsilon_{i j} x^{j} .
$$

This expression shows that the Nappi-Witten background indeed describes a plane fronted gravitational wave with rather simple (constant) polarization.

To define the action of the Nappi-Witten string properly we add extra coordinates, $x^{A}$, describing $d$ flat Euclidean dimensions, to the four ones already present in eq.(3):

$$
\begin{aligned}
S= & \frac{1}{2 \pi} \int d \tau d \sigma\left[\partial_{a} x^{A} \partial^{a} x^{A}+\frac{k}{2}\left(\partial_{a} x^{i} \partial^{a} x^{i}-2 \partial_{a} U \partial^{a} V+\left(b-\left(x^{i}\right)^{2} / 4\right) \partial_{a} U \partial^{a} U\right.\right. \\
& \left.\left.-\epsilon_{i j} x^{j} \partial_{a} x^{i} \partial_{b} U \epsilon^{a b}\right)\right] .
\end{aligned}
$$

In eq.(4) we fixed the conformal gauge on the world sheet: $h_{a b}=\eta_{a b},(a=\tau, \sigma)$, with $\eta_{\tau \tau}=-\eta_{\sigma \sigma}=1$ and also set the the slope parameter, $\alpha^{\prime}=1 / 2$, in spite of considering the case of closed rather than open strings. In the $x^{i}, U, V$ part of this action we wrote explicitly the $k$ parameter of the original Nappi Witten action.

The equations of motion following from eq.(4) are

$$
\begin{gathered}
\left(\partial_{\tau}^{2}-\partial_{\sigma}^{2}\right) x^{A}=0 \\
\left(\partial_{\tau}^{2}-\partial_{\sigma}^{2}\right) U=0 \\
2\left(\partial_{\tau}^{2}-\partial_{\sigma}^{2}\right) x^{i}+\frac{x^{i}}{2} \partial_{a} U \partial^{a} U+2 \epsilon^{j i}\left(\partial_{\tau} x^{j} \partial_{\sigma} U-\partial_{\sigma} x^{j} \partial_{\tau} U\right)=0 \\
-2\left(\partial_{\tau}^{2}-\partial_{\sigma}^{2}\right) V+2 \partial_{a}\left[\left(b-\frac{\left(x^{i}\right)^{2}}{4}\right) \partial^{a} U\right]+2 \epsilon^{i j} \partial_{\tau} x^{j} \partial_{\sigma} x^{i}=0 .
\end{gathered}
$$

These equations are supplemented by the vanishing of the world sheet energy momentum tensor, $T_{a b}$, expressing the reparametrization invariance of the string action.

The light-cone gauge on the target space is fixed by setting $U=P \tau$, which obviously solves the free equation for $U$ in (5). The equations of motion for the $x^{i}$ coordinates simplify in this gauge:

$$
2\left(\partial_{\tau}^{2}-\partial_{\sigma}^{2}\right) x^{i}+\frac{x^{i}}{2} P^{2}+2 P \epsilon^{i j} \partial_{\sigma} x^{j}=0
$$


while the $x^{A}$ still has to satisfy the free equations appearing in (5). The two independent components of the constraint $T_{a b}=0$ take the form

$$
\begin{aligned}
& P \partial_{\tau} V=\frac{1}{2}\left[\left(\partial_{\tau} x^{i}\right)^{2}+\left(\partial_{\sigma} x^{i}\right)^{2}+P^{2}\left(b-\frac{\left(x^{i}\right)^{2}}{4}\right)+\frac{2}{k}\left(\left(\partial_{\tau} x^{A}\right)^{2}+\left(\partial_{\sigma} x^{A}\right)^{2}\right)\right], \\
& P \partial_{\sigma} V=\partial_{\tau} x^{i} \partial_{\sigma} x^{i}+\frac{2}{k} \partial_{\tau} x^{A} \partial_{\sigma} x^{A} .
\end{aligned}
$$

If $P \neq 0$ we use the two equations appearing in (7) to determine the second light-cone coordinate, $V$, in terms of $x^{i}$ and $x^{A}$. Indeed one readily verifies, that $V$ determined this way satisfies not just the integrability condition, but also the equation of motion in (5) whenever $x^{i}\left(x^{A}\right)$ satisfy their equation of motion, eq.(6) (eq.(5) respectively). Since all fields are periodic in the case of closed strings, integrating the second equation in (7) over $\sigma$ we get the usual constraint

$$
\int_{0}^{2 \pi} d \sigma\left(\partial_{\tau} x^{i} \partial_{\sigma} x^{i}+\frac{2}{k} \partial_{\tau} x^{A} \partial_{\sigma} x^{A}\right)=0
$$

which enforces invariance under shifting $\sigma$ by a constant.

From the light-cone form of the action

$$
\begin{aligned}
S= & \frac{1}{2 \pi} \int d \tau d \sigma\left[\partial_{a} x^{A} \partial^{a} x^{A}+\frac{k}{2}\left(\partial_{a} x^{i} \partial^{a} x^{i}-2 P \partial_{\tau} V+\left(b-\left(x^{i}\right)^{2} / 4\right) P^{2}\right.\right. \\
& \left.\left.+P \epsilon_{i j} x^{j} \partial_{\sigma} x^{i}\right)\right]
\end{aligned}
$$

one readily derives the canonical momenta

$$
\Pi^{i}=\frac{1}{\pi} \frac{k}{2} \partial_{\tau} x^{i}, \quad \Pi^{A}=\frac{1}{\pi} \partial_{\tau} x^{A}
$$

and constructs the canonical Hamiltonian in the usual way:

$$
\begin{aligned}
H= & \int_{0}^{2 \pi} \frac{d \sigma}{2 \pi}\left[(\pi)^{2}\left(\Pi^{A}\right)^{2}+\left(\partial_{\sigma} x^{A}\right)^{2}+\frac{2}{k}(\pi)^{2}\left(\Pi^{i}\right)^{2}\right. \\
+ & \left.\frac{k}{2}\left(\left(\partial_{\sigma} x^{i}\right)^{2}-\left(b-\left(x^{i}\right)^{2} / 4\right) P^{2}-P \epsilon_{i j} x^{j} \partial_{\sigma} x^{i}\right)\right] \\
= & \int_{0}^{2 \pi} \frac{d \sigma}{2 \pi}\left[\left(\partial_{\tau} x^{A}\right)^{2}+\left(\partial_{\sigma} x^{A}\right)^{2}+\frac{k}{2}\left(\left(\partial_{\tau} x^{i}\right)^{2}+\left(\partial_{\sigma} x^{i}\right)^{2}-\left(b-\left(x^{i}\right)^{2} / 4\right) P^{2}\right.\right. \\
& \left.\left.-P \epsilon_{i j} x^{j} \partial_{\sigma} x^{i}\right)\right] .
\end{aligned}
$$




\section{$\underline{\text { 2. Global symmetries }}$}

The symmetries of the target space appear in string theory as global (world sheet independent) internal symmetries. Since they play an essential role in the quantization and in the interpretation of the results, we describe them in some details. For the theory described by eq.(4) there are three types of global symmetries: the ones related to the four "interesting" coordinates of the Nappi Witten solution, the ones belonging to the $x^{A}$ part of the action and the ones mixing these two types of coordinates. We are mainly interested in the first and third types of these global symmetries since the symmetries of the $x^{A}$ part form the standard $E_{d}$. A particularly interesting question is the possible reduction of these symmetries when one moves to the light-cone gauge; i.e. when one replaces eq.(4) by eq.(9).

As for the first type of global symmetries we note that the Lagrangian in eq.(4) is obviously invariant under the (infinitesimal) translation of the $U$ or the $V$ coordinates:

$$
\begin{array}{ll}
T_{u_{0}}: & x^{i} \rightarrow x^{i} ; \quad U \rightarrow U+u_{0} ; \quad V \rightarrow V, \\
T_{v_{0}}: & x^{i} \rightarrow x^{i} ; \quad U \rightarrow U ; \quad V \rightarrow V+v_{0},
\end{array}
$$

while it changes by a total divergence under the rotation of the $x^{i}$ coordinates:

$$
T_{\alpha}: \quad x^{i} \rightarrow x^{i}+\alpha \epsilon^{i j} x_{j} ; \quad U \rightarrow U ; \quad V \rightarrow V
$$

A direct computation shows that the action in eq.(4) is also invariant under the four "twisted translations" of the $x^{i}$ coordinates:

$$
\begin{array}{cll}
T_{\delta_{1}}: & x_{1} \rightarrow x_{1}+\delta_{1} \cos (U / 2) ; \quad x_{2} \rightarrow x_{2}-\delta_{1} \sin (U / 2) ; \quad U \rightarrow U ; \\
V & \rightarrow V-\left(\delta_{1} / 2\right)\left(x_{1} \sin (U / 2)+x_{2} \cos (U / 2)\right), & \\
T_{\delta_{2}}: & x_{1} \rightarrow x_{1}+\delta_{2} \sin (U / 2) ; \quad x_{2} \rightarrow x_{2}+\delta_{2} \cos (U / 2) ; \quad U \rightarrow U ; \\
V & \rightarrow V-\left(\delta_{2} / 2\right)\left(x_{2} \sin (U / 2)-x_{1} \cos (U / 2)\right), \\
T_{\Delta_{1}}: & x_{1} \rightarrow x_{1}+\Delta_{1} \cos (U / 2) ; \quad x_{2} \rightarrow x_{2}+\Delta_{1} \sin (U / 2) ; \quad U \rightarrow U ; \\
V & \rightarrow V-\left(\Delta_{1} / 2\right)\left(x_{1} \sin (U / 2)-x_{2} \cos (U / 2)\right), & \\
T_{\Delta_{2}}: & x_{1} \rightarrow x_{1}-\Delta_{2} \sin (U / 2) ; \quad x_{2} \rightarrow x_{2}+\Delta_{2} \cos (U / 2) ; \quad U \rightarrow U ; \\
V & \rightarrow V-\left(\Delta_{2} / 2\right)\left(x_{2} \sin (U / 2)+x_{1} \cos (U / 2)\right) .
\end{array}
$$

Using these infinitesimal transformations one can compute the Lie algebra of the 7 parameter symmetry group, $M_{7}$. We find that the two types of translations commute with each other, while among themselves they yield the $V$ translation:

$$
\left[T_{\delta_{i}}, T_{\Delta_{j}}\right]=0, \quad\left[T_{\delta_{2}}, T_{\delta_{1}}\right]=-\left[T_{\Delta_{2}}, T_{\Delta_{1}}\right]=T_{v_{0}}
$$


Both the rotation and the $U$ translation act on the $x^{i}$ translations:

$$
\begin{gathered}
{\left[T_{\alpha}, T_{\delta_{i}}\right]=-\epsilon_{i j} T_{\delta_{j}}, \quad\left[T_{u_{0}}, T_{\delta_{i}}\right]=\frac{1}{2} \epsilon_{i j} T_{\delta_{j}},} \\
{\left[T_{\alpha}, T_{\Delta_{i}}\right]=-\epsilon_{i j} T_{\Delta_{j}}, \quad\left[T_{u_{0}}, T_{\Delta_{i}}\right]=-\frac{1}{2} \epsilon_{i j} T_{\Delta_{j}} .}
\end{gathered}
$$

It is easy to see from eq.(13-14) that one can form two independent combinations of the rotation and $U$ translation, $\left(T_{u_{0}} \pm T_{\alpha} / 2\right)$, that act only on one type of the $x^{i}$ translations, leaving the other type invariant:

$$
\begin{aligned}
{\left[T_{u_{0}}+T_{\alpha} / 2, T_{\delta_{i}}\right] } & =0, \quad\left[T_{u_{0}}+T_{\alpha} / 2, T_{\Delta_{i}}\right]=-\epsilon_{i j} T_{\Delta_{j}} \\
{\left[T_{u_{0}}-T_{\alpha} / 2, T_{\Delta_{i}}\right] } & =0, \quad\left[T_{u_{0}}-T_{\alpha} / 2, T_{\delta_{i}}\right]=\epsilon_{i j} T_{\delta_{j}} .
\end{aligned}
$$

These linear combinations, together with the "affected" translations and the $V$ translation form two (chiral) copies of the $E_{2}^{c}$ algebra inherent in the NappiWitten construction ${ }^{\dagger}$.

The conserved currents belonging to these symmetries are derived in the usual way; we list only some of them:

$$
\begin{aligned}
J_{a}^{\delta_{1}} & =\frac{k}{4 \pi}\left[\left(2 \partial_{a} x^{1}-x^{2} \partial_{b} U \epsilon^{a b}\right) \cos \left(\frac{U}{2}\right)-\left(2 \partial_{a} x^{2}+x^{1} \partial_{b} U \epsilon^{a b}\right) \sin \left(\frac{U}{2}\right)\right. \\
& +\partial_{a} U\left(\left(x_{1} \sin \left(\frac{U}{2}\right)+x_{2} \cos \left(\frac{U}{2}\right)\right)\right] \\
J_{a}^{v_{0}} & =-\frac{k}{2 \pi} \partial_{a} U \\
J_{a}^{u_{0}} & =\frac{k}{4 \pi}\left[-2 \partial_{a} V+2\left(b-\frac{\left(x^{i}\right)^{2}}{4}\right) \partial_{a} U-\epsilon^{i j} \epsilon^{b a} x^{j} \partial_{b} x^{i}\right] \\
J_{a}^{\alpha} & =\frac{k}{4 \pi}\left[2 \partial_{a} x^{i}-\epsilon^{i j} x_{j} \partial_{b} U \epsilon^{a b}\right] \epsilon^{i m} x_{m} .
\end{aligned}
$$

Integrating the $\tau$ components of these currents over $\sigma$ we get the conserved charges belonging to the various symmetries. It is interesting to notice, that the currents belonging to the various $x^{i}$ translations are linear in $x^{i}$, and it is only the current of the $U$ translation that depends on the second light-cone coordinate, $V$.

Comparing the light cone form of the action, eq.(9), and the covariant one in eq.(4), we see that the transformations generating the first type of global symmetries of eq.(4) - with the exception of the $U$ translations - are also explicit symmetries of eq.(9). This situation is significantly different from the case of

$\dagger$ C. Duval [unpublished] has shown that this seven parameter symmetry is indeed the full isometry of the metric (3). 
strings propagating on flat Minkowski space, when going to the light cone gauge reduces the explicit $S O(D-1,1) \triangleleft T^{D}$ symmetry of the covariant action to a manifest $S O(D-2) \triangleleft T^{D-2}$ only.

In the light-cone gauge, where $\partial_{\tau} U=P, \partial_{\sigma} U=0$, eq.(15) simplifies slightly. We find trivially $Q^{v_{0}}=-k P$, while a short computation using eq.(7) and eq.(11) yields:

$$
Q^{u_{0}} \equiv \int_{0}^{2 \pi} d \sigma J_{\tau}^{u_{0}}=-\frac{1}{P} H .
$$

This shows that all seven charges of the covariant action, that belong to the first type of global symmetries, are also conserved in the light cone gauge.

In the quantum theory (or in the classical one using the Poisson brackets) these charges satisfy an algebra similar to the one given by eq.(12-14). This algebra has three independent Casimir operators, that can be chosen in the following way: a linear one $C_{1}=Q^{v_{0}}$, and two quadratic ones

$$
\begin{aligned}
& C_{2}=4 Q^{u_{0}} Q^{v_{0}}-\left(Q^{\delta_{i}}\right)^{2}-\left(Q^{\Delta_{i}}\right)^{2}, \\
& C_{3}=2 Q^{\alpha} Q^{v_{0}}+\left(Q^{\delta_{i}}\right)^{2}-\left(Q^{\Delta_{i}}\right)^{2} .
\end{aligned}
$$

In terms of the chiral $E_{2}^{c}$ algebra $C_{2}$ correponds to the sum while $C_{3}$ to the difference of the left and right Casimirs.

The symmetries of the covariant action mixing the "Nappi Witten" and the $x^{A}$ coordinates are generated by the infinitesimal transformations:

$$
T_{A}^{\beta}: \quad x^{A} \rightarrow x^{A}+\frac{k}{2} \beta U, \quad x^{B} \rightarrow x^{B} B \neq A, \quad U \rightarrow U, \quad V \rightarrow V+\beta x^{A} .
$$

(Here $\beta$ is an infinitesimal parameter). Using the infinitesimal transformations of the $E_{d}$ symmetry:

$$
\begin{aligned}
T_{A}: & x^{A} \rightarrow x^{A}+\delta^{A}, \quad U \rightarrow U, \quad V \rightarrow V, \\
T_{A B}: & x^{C} \rightarrow x^{C}+\gamma\left(\delta^{A C} x^{B}-\delta^{B C} x^{A}\right), \quad U \rightarrow U, \quad V \rightarrow V,
\end{aligned}
$$

one readily proves that

$$
\left[T_{A}^{\beta}, T_{u_{0}}\right]=\frac{k}{2} T_{A}, \quad\left[T_{A}^{\beta}, T_{B}\right]=\delta_{A B} T_{v_{0}}, \quad\left[T_{A B}, T_{C}^{\beta}\right]=\delta_{B C} T_{A}^{\beta}-\delta_{A C} T_{B}^{\beta} .
$$

Furthermore it is easy to see that the symmetries generated by $T_{A}^{\beta}$ are also explicit symmetries of the light cone action, eq.(9). The conserved current belonging to the $T_{A}^{\beta}$ transformation has the form:

$$
J_{a}^{\beta}=\frac{k}{2 \pi}\left(\partial_{a} x^{A} U-x^{A} \partial_{a} U\right)
$$


thus in the light cone gauge the conserved charge associated to it becomes

$$
Q_{A}^{\beta}=-\frac{k P}{2 \pi} \int_{0}^{2 \pi} d \sigma\left(\tau \partial_{\tau} x^{A}-x^{A}\right) .
$$

\section{Oscillator expansion and quantization}

In the light-cone gauge the equation of motion for the $x^{i}$ fields, eq.(6), is not only linear in the $x^{i}$-s but contains only constant coefficients. Therefore decomposing the $x^{i}$ fields into Fourier modes

$$
x^{i}(\tau, \sigma)=\sum_{n} X_{n}^{i}(\tau) e^{i n \sigma}
$$

we find that the Fourier coefficients, $X_{n}^{i}(i=1,2)$, satisfy coupled oscillator type equations for all $n$ :

$$
\begin{aligned}
& 2 \frac{d^{2} X_{n}^{1}}{d \tau^{2}}+\left(2 n^{2}+\frac{P^{2}}{2}\right) X_{n}^{1}+2 \operatorname{Pin} X_{n}^{2}=0 \\
& 2 \frac{d^{2} X_{n}^{2}}{d \tau^{2}}+\left(2 n^{2}+\frac{P^{2}}{2}\right) X_{n}^{2}-2 \operatorname{Pin} X_{n}^{1}=0
\end{aligned}
$$

Making the ansatz $X_{n}^{i}=C_{n}^{i} e^{i \omega_{n} \tau}$ we find

$$
\omega_{n}^{2}=\left(n \pm \frac{P}{2}\right)^{2} .
$$

Either directly from eq.(6), or from eq.(19-20), we see that the "zero modes" $(n=$ 0 ), that describe the motion of the string's center of mass, also satisfy oscillator equations. This behaviour is strikingly different from the usual, uniform motion of the CM coordinate in flat dimensions. The frequency of these CM oscillations is $P / 2$; this precisely corresponds to the coefficient of the quadratic potential in eq. (9).

The interesting property of the $\omega_{n}= \pm|n \pm P / 2|$ eigenfrequencies (we need all four combinations!) is that substituting them into eq.(18) we get 'almost' chiral expressions. Indeed, when $0<P / 2<1$, we find - having carefully determined the eigen-directions of the matrix originating from eq.(19) - that:

$$
\begin{aligned}
x^{1}+i x^{2} & =\frac{1}{\sqrt{k}}\left[\frac{2}{P} K^{\dagger} e^{i \frac{P}{2} \tau}+\frac{2}{P} \Lambda e^{-i \frac{P}{2} \tau}+e^{i \frac{P}{2} \tau} \sum_{n>0}\left(\frac{\lambda_{n}^{+}}{n-P / 2} e^{-i n(\tau+\sigma)}\right.\right. \\
& \left.+\frac{\left(k_{n}^{+}\right)^{\dagger}}{n+P / 2} e^{i n(\tau+\sigma)}\right)+e^{-i \frac{P}{2} \tau} \sum_{n>0}\left(\frac{\lambda_{n}^{-}}{n+P / 2} e^{-i n(\tau-\sigma)}\right. \\
& \left.\left.+\frac{\left(k_{n}^{-}\right)^{\dagger}}{n-P / 2} e^{i n(\tau-\sigma)}\right)\right]
\end{aligned}
$$


and

$$
\begin{aligned}
x^{1}-i x^{2} & =\frac{1}{\sqrt{k}}\left[\frac{2}{P} K e^{-i \frac{P}{2} \tau}+\frac{2}{P}(\Lambda)^{\dagger} e^{i \frac{P}{2} \tau}+e^{-i \frac{P}{2} \tau} \sum_{n>0}\left(\frac{\left(\lambda_{n}^{+}\right)^{\dagger}}{n-P / 2} e^{i n(\tau+\sigma)}\right.\right. \\
& \left.+\frac{k_{n}^{+}}{n+P / 2} e^{-i n(\tau+\sigma)}\right)+e^{i \frac{P}{2} \tau} \sum_{n>0}\left(\frac{\left(\lambda_{n}^{-}\right)^{\dagger}}{n+P / 2} e^{i n(\tau-\sigma)}\right. \\
& \left.\left.+\frac{k_{n}^{-}}{n-P / 2} e^{-i n(\tau-\sigma)}\right)\right] .
\end{aligned}
$$

Here the various normalizations are introduced for later convenience and for the classical Fourier coefficients $\nmid$ stands for complex conjugation. The distribution of complex conjugated quantities in eq.(21-22) guaranties that $\left(x^{1}+i x^{2}\right)^{\dagger}=$ $x^{1}-i x^{2}$.

We quantize our $x^{i}$ fields by promoting the various Fourier components appearing in eq.(21-22) to operators. We fix their commutators by requiring, that the canonical equal time commutation relations

$$
\begin{aligned}
{\left[\Pi^{i}(\tau, \sigma), x^{j}\left(\tau, \sigma^{\prime}\right)\right] } & =-i \delta^{i j} \delta\left(\sigma-\sigma^{\prime}\right), \\
{\left[\Pi^{i}(\tau, \sigma), \Pi^{j}\left(\tau, \sigma^{\prime}\right)\right] } & =0, \quad\left[x^{i}(\tau, \sigma), x^{j}\left(\tau, \sigma^{\prime}\right)\right]=0,
\end{aligned}
$$

hold when $\Pi^{i}(\tau, \sigma)$ is expressed in terms of $x^{i}$, as in eq.(10). Proceeding this way we find that the only nonvanishing commutators for the non zero modes (i.e. $n, m>0$ ) are the following:

$$
\begin{array}{ll}
{\left[k_{n}^{+},\left(k_{m}^{+}\right)^{\dagger}\right]=\delta_{n, m}(n+P / 2),} & {\left[\lambda_{n}^{+},\left(\lambda_{m}^{+}\right)^{\dagger}\right]=\delta_{n, m}(n-P / 2),} \\
{\left[k_{n}^{-},\left(k_{m}^{-}\right)^{\dagger}\right]=\delta_{n, m}(n-P / 2),} & {\left[\lambda_{n}^{-},\left(\lambda_{m}^{-}\right)^{\dagger}\right]=\delta_{n, m}(n+P / 2),}
\end{array}
$$

while for the zero mode operators

$$
\left[\Lambda,(\Lambda)^{\dagger}\right]=P / 2 ; \quad\left[K,(K)^{\dagger}\right]=P / 2
$$

results. Eq.(23) shows, that in both light-cone directions we have a set of $n+P / 2$ modded and a set of $n-P / 2$ modded oscillators. Eq.(24) implies that the oscillatory nature of the CM coordinate's motion persists to the quantum theory.

The appearance of these non-integrally modded ("twisted") oscillators can be understood in the following way: Rewriting eq.(6) as:

$$
\square\left(x^{1} \pm i x^{2}\right)+\frac{P^{2}}{4}\left(x^{1} \pm i x^{2}\right) \mp i P \partial_{\sigma}\left(x^{1} \pm i x^{2}\right)=0,
$$

and introducing the complex $\Phi(\tau, \sigma)$ field through the ansatz:

$$
x^{1}+i x^{2}=e^{-i \frac{P}{2} \sigma} \Phi(\tau, \sigma) ; \quad x^{1}-i x^{2}=e^{i \frac{P}{2} \sigma} \Phi^{\dagger}(\tau, \sigma)
$$


one easily deduces that $\Phi$ obeys the free field equation: $\square \Phi=0$. Thus $x^{1} \pm i x^{2}$ can be written as:

$x^{1}+i x^{2}=e^{-i \frac{P}{2} \sigma}\left(\phi(\tau-\sigma)+\psi^{\dagger}(\tau+\sigma)\right) ; \quad x^{1}-i x^{2}=e^{i \frac{P}{2} \sigma}\left(\phi^{\dagger}(\tau-\sigma)+\psi(\tau+\sigma)\right)$.

However, because of the $\exp ( \pm i P \sigma / 2)$ prefactor, the free fields, $\phi(\tau-\sigma)$ and $\psi(\tau+\sigma)$, must be "twisted" (i.e. must satisfy non-periodic boundary conditions in $\sigma)$ to yield periodic $x^{1} \pm i x^{2}$-s.

Since the equations of motion for the $x^{A}$ coordinates are the standard ones we get the standard oscillator expansion for them:

$$
x^{A}=q^{A}+\frac{p^{A}}{2} \tau+\frac{i}{2} \sum_{n \neq 0}\left(\frac{\alpha_{n}^{-A}}{n} e^{-i n(\tau-\sigma)}+\frac{\alpha_{n}^{+A}}{n} e^{-i n(\tau+\sigma)}\right) .
$$

\section{Hamilton operator, $M^{2}$ and physical states}

Using the oscillator expansions eq. $(21,22,25)$ in eq.(11) we find the Hamilton operator:

$$
\begin{aligned}
H & =\frac{1}{4}\left(p^{A}\right)^{2}+\sum_{n>0}\left(\alpha_{-n}^{-A} \alpha_{n}^{-A}+\alpha_{-n}^{+A} \alpha_{n}^{+A}\right) \\
& +\sum_{n>0}\left(\left(\lambda_{n}^{+}\right)^{\dagger} \lambda_{n}^{+}+\left(\lambda_{n}^{-}\right)^{\dagger} \lambda_{n}^{-}+\left(k_{n}^{+}\right)^{\dagger} k_{n}^{+}+\left(k_{n}^{-}\right)^{\dagger} k_{n}^{-}\right) \\
& +(K)^{\dagger} K+(\Lambda)^{\dagger} \Lambda-\frac{k}{2} b P^{2}+a .
\end{aligned}
$$

Here $a$ is a normal ordering constant, that can be determined by $\zeta$ function regularization since it originates in reordering the integer modded oscillators, the zero mode ones and the $n \pm P / 2$ modded ones:

$$
\begin{aligned}
a & =2 d \frac{1}{2}\left(-\frac{1}{12}\right)+2 \frac{1}{2} \frac{P}{2}+2 \frac{1}{2}\left(\sum_{n=1}^{\infty}(n+P / 2)+\sum_{n=1}^{\infty}(n-P / 2)\right) \\
& =\left(-\frac{d}{12}\right)+\frac{P}{2}+\zeta(-1, P / 2)+1-P / 2+\zeta(-1,1-P / 2) \\
& =\left(-\frac{d+2}{12}\right)+1+\frac{P}{2}\left(1-\frac{P}{2}\right)
\end{aligned}
$$

From eq.(26-27) one can read off the spectrum, but to analyse it we have to determine what are the restrictions on the physical states. Also to put the spectrum of the Hamiltonian into an appropriate perspective we have to derive the analog of the $M^{2}$ operator for the gravitational background described in eq. (4). 
In the usual case, when strings propagate on a flat Minkowski background, the $M^{2}$ operator is nothing but the quadratic Casimir of the symmetry group constructed of the generators of translations. Therefore in the present case the appropriate generalisation is to consider a linear combination of $C_{2}$ and $\left(p^{A}\right)^{2}$. The relative coefficient between them is determined from requiring it to reproduce the well known result in the limiting case of flat Minkowski space:

$$
M^{2}=\frac{1}{k}\left(4 Q^{u_{0}} Q^{v_{0}}-\left(Q^{\delta_{i}}\right)^{2}-\left(Q^{\Delta_{i}}\right)^{2}\right)-p^{A} p^{A} .
$$

Since the charges $Q^{\delta_{i}}, Q^{\Delta_{i}}$, contain only the zero mode operators (e.g. $Q^{\delta_{1}}=$ $\left.i \sqrt{k}\left(\Lambda^{\dagger}-\Lambda\right), Q^{\delta_{2}}=-\sqrt{k}\left(\Lambda^{\dagger}+\Lambda\right)\right)$, when expressed in terms of the oscillators, $M^{2}$ has the form:

$$
\begin{aligned}
M^{2} & =4\left[\sum_{n>0}\left(\alpha_{-n}^{-A} \alpha_{n}^{-A}+\alpha_{-n}^{+A} \alpha_{n}^{+A}\right)\right. \\
& +\sum_{n>0}\left(\left(\lambda_{n}^{+}\right)^{\dagger} \lambda_{n}^{+}+\left(\lambda_{n}^{-}\right)^{\dagger} \lambda_{n}^{-}+\left(k_{n}^{+}\right)^{\dagger} k_{n}^{+}+\left(k_{n}^{-}\right)^{\dagger} k_{n}^{-}\right) \\
& \left.-\frac{k}{2} b P^{2}+a-P / 2\right] .
\end{aligned}
$$

This expression also shows, that the zero mode operators, that are present in $H$, disappear from $M^{2}$.

The physical states are restricted by the left-right symmetry condition: i.e. eq.(8) imposes on them the invariance under shifting the $\sigma$ coordinate. Carrying out the integration in eq.(8) we get:

$$
\begin{aligned}
& \sum_{n>0}\left[\alpha_{-n}^{-A} \alpha_{n}^{-A}+\frac{n}{n+P / 2}\left(\lambda_{n}^{-}\right)^{\dagger} \lambda_{n}^{-}+\frac{n}{n-P / 2}\left(k_{n}^{-}\right)^{\dagger} k_{n}^{-}\right] \\
& -\sum_{n>0}\left[\alpha_{-n}^{+A} \alpha_{n}^{+A}+\frac{n}{n-P / 2}\left(\lambda_{n}^{+}\right)^{\dagger} \lambda_{n}^{+}+\frac{n}{n+P / 2}\left(k_{n}^{+}\right)^{\dagger} k_{n}^{+}\right]=0 .
\end{aligned}
$$

Two things must be remarked about this equation that generalizes the usual left right symmetry condition: it requires that the total excitation numbers of the " $n$ index" oscillators be the same for the + and - light cone directions, irrespective of how these numbers are distributed among the $n, n+P / 2$ and $n-P / 2$ modded ones. In the same time eq.(30) says nothing about the zero mode oscillators. The absence of the zero mode operators in $M^{2}$ and in eq.(30) implies, that physical states with the same $M^{2}$ are infinitely degenerate. Indeed denoting the excitation numbers of the $\Lambda$ and $K$ oscillators by $n_{\Lambda}$ and $n_{K}$ respectively and making the replacements

$$
\tilde{n}_{\Lambda}=n_{\Lambda}+N, \quad \tilde{n}_{K}=n_{K}+M \quad(N, M>0)
$$


generates new physical states from the old ones. These new states correspond to placing the string as a whole - no matter how it is "wiggling" - on the various discrete energy levels in the external harmonic oscillator potential.

Using eq.(20,21, and 25) in eq.(15) to compute $Q^{\alpha}$ we get:

$$
\begin{aligned}
Q^{\alpha}=\frac{2}{P}\left(K^{\dagger} K-\Lambda^{\dagger} \Lambda\right) & -\sum_{n>0}\left[\frac{1}{n+P / 2}\left[\left(k_{n}^{+}\right)^{\dagger} k_{n}^{+}-\left(\lambda_{n}^{-}\right)^{\dagger} \lambda_{n}^{-}\right]\right. \\
& \left.+\frac{1}{n-P / 2}\left[\left(k_{n}^{-}\right)^{\dagger} k_{n}^{-}-\left(\lambda_{n}^{+}\right)^{\dagger} \lambda_{n}^{+}\right]\right] .
\end{aligned}
$$

Using this expression one can write the new left right symmetry condition, eq.(30), in an alternative form:

$$
\begin{aligned}
& \sum_{n>0}\left[\alpha_{-n}^{-A} \alpha_{n}^{-A}+\left(\lambda_{n}^{-}\right)^{\dagger} \lambda_{n}^{-}+\left(k_{n}^{-}\right)^{\dagger} k_{n}^{-}\right]+K^{\dagger} K \\
& -\sum_{n>0}\left[\alpha_{-n}^{+A} \alpha_{n}^{+A}+\left(\lambda_{n}^{+}\right)^{\dagger} \lambda_{n}^{+}+\left(k_{n}^{+}\right)^{\dagger} k_{n}^{+}\right]-\Lambda^{\dagger} \Lambda-\frac{P}{2} Q^{\alpha}=0 .
\end{aligned}
$$

\section{$\underline{5 .}$ Critical dimension and the chiral algebra}

The next step is to try to derive the critical dimension and the intercept (i.e. the normal ordering constant $a$ ) in the light cone gauge. In the case of strings on flat Minkowski space these numbers are obtained by requiring the complete $S O(D-1,1)$ symmetry algebra of the covariant action to hold even in the light cone formalism where only its $S O(D-2)$ part is a manifest symmetry. As we mentioned earlier for the Nappi Witten string it is only the $U$ translation that is not an explicit symmetry of the light cone action, eq.(9). Therefore the only thing we can impose is the vanishing of the commutators between $Q^{u_{0}}$ and those charges that depend non-linearly on the oscillators, i.e. the vanishing of $\left[Q^{u_{0}}, Q^{\alpha}\right]$ and $\left[Q^{u_{0}}, M^{A B}\right]$, where

$$
M^{A B}=\frac{1}{2 \pi} \int_{0}^{2 \pi} d \sigma\left(x^{A} \partial_{\tau} x^{B}-x^{B} \partial_{\tau} x^{A}\right) .
$$

However a simple computation, using the explicit form of these charges, shows that these commutators vanish without imposing any restriction either on $d$ or on $a$. One can also check that all commutators of the charges $Q_{A}^{\beta}=-k P q^{a}$, belonging to the symmetries mixing the "Nappi Witten" coordinates and the $x^{A}$ ones, are the required ones independently of the values of $d$ and $a$. Therefore the critical dimension is not determined in this light cone quantization of the 
Nappi Witten string. Though surprising, this conclusion is in accord with what was found in ref.[2] among somewhat similar circumstances. ${ }^{\dagger}$

It is also interesting to determine how the $\hat{E}_{2}^{c}$ chiral Kac Moody algebra of the Nappi Witten construction gets distorted in the light cone gauge. In the covariant formalism the components of the holomorphic current, $\partial g g^{-1}$, $\left(\partial=\partial_{\tau}-\partial_{\sigma}\right)$ that belong to the various generators of $E_{2}^{c}$ in the $g=\exp \left[a_{1} P_{1}+\right.$ $\left.a_{2} P_{2}\right] \exp [U J-V T]$ parametrization, are given by $[3,4]$ :

$$
\begin{aligned}
P_{1}\left(\xi_{-}\right) & \equiv J^{1}\left(\xi_{-}\right)=k\left(\partial a_{1}+a_{2} \partial U\right) \\
P_{2}\left(\xi_{-}\right) & \equiv J^{2}\left(\xi_{-}\right)=k\left(\partial a_{2}-a_{1} \partial U\right) \\
J\left(\xi_{-}\right) & \equiv J^{3}\left(\xi_{-}\right)=k\left(-\partial V+\frac{1}{2} \epsilon^{i j} a_{i} \partial a_{j}+\left(b-\frac{1}{2} a_{i} a_{i}\right) \partial U\right) \\
T\left(\xi_{-}\right) & \equiv J^{4}\left(\xi_{-}\right)=k \partial U .
\end{aligned}
$$

Here we introduced $\xi_{-}=\tau-\sigma$ and also define the $J_{n}^{k}$ Fourier coefficients of $J^{k}\left(\xi_{-}\right) k=1, . .4$ as

$$
J^{k}\left(\xi_{-}\right)=\sum_{-\infty}^{\infty} J_{n}^{k} e^{-i n \xi_{-}}
$$

Using the inverse of eq.(2) it is easy to express $J^{k}$ in terms of our $x^{i}$ fields. The fact that in the light cone gauge the Kac Moody algebra generated by $J_{n}^{k}$ gets distorted can be seen already from the defining relation, $U=P \tau$, since this effectively sets $J_{n}^{4}=k P \delta_{n, 0}$. Nevertheless a straightforward computation using the inverse of eq.(2) and the oscillator expansions in eq. $(21,22)$ yields $(n>0)$ :

$$
\begin{array}{cl}
J_{0}^{1}=i \sqrt{k}\left(\Lambda^{\dagger}-\Lambda\right) & J_{n}^{1}=-i \sqrt{k}\left(\lambda_{n}^{-}+k_{n}^{-}\right) \quad J_{-n}^{1}=i \sqrt{k}\left(\left(\lambda_{n}^{-}\right)^{\dagger}+\left(k_{n}^{-}\right)^{\dagger}\right) \\
J_{0}^{2}=-\sqrt{k}\left(\Lambda^{\dagger}+\Lambda\right) & J_{n}^{2}=\sqrt{k}\left(k_{n}^{-}-\lambda_{n}^{-}\right) \quad J_{-n}^{2}=\sqrt{k}\left(\left(k_{n}^{-}\right)^{\dagger}-\left(\lambda_{n}^{-}\right)^{\dagger}\right) .
\end{array}
$$

Now one easily obtaines on account of eq. $(22,23)$ that for any $n, m \in Z$

$$
\left[J_{n}^{i}, J_{m}^{j}\right]=2 k n \delta_{n+m}^{i j}+i \epsilon^{i j} k P \delta_{n+m} \quad i, j=1,2 .
$$

Eq.(36) shows that the Kac Moody subalgebra generated by $J_{n}^{1}, J_{n}^{2}$ and $J_{n}^{4}$ remains unbroken even in the light cone gauge. The computation of $J_{n}^{3}$ - that exploits the constraints in eq. $(7)$ - is more involved and we give here only the final result expressed in terms of the Fourier coefficients of $J_{n}^{1}, J_{n}^{2}$ and $x^{A}$ :

$$
J_{n}^{3}=-\frac{1}{P}\left(\frac{1}{2 k}\left(:\left(J^{1}\right)^{2}:_{n}+:\left(J^{2}\right)^{2}:_{n}\right)+\sum_{l}: \tilde{\alpha}_{l}^{-A} \tilde{\alpha}_{n-l}^{-A}:+\left(-\frac{k}{2} b P^{2}+\hat{a}\right) \delta_{n, 0}\right),
$$

$\dagger$ The critical dimension in the light-cone quantization of $2 D$ sigma models was derived recently in ref.[11], using the functional integral formalism. 
where $\tilde{\alpha}_{0}^{-A}=p^{A} / 2, \tilde{\alpha}_{k}^{-A}=\alpha_{k}^{-A}$ if $k \neq 0,:$ denotes the standard normal ordering (i.e. creation operators stand to the left of annihilation ones) and $\hat{a}$ is a normal ordering constant. Eq.(37) leads to the commutators:

$$
\left[J_{l}^{3}, J_{m}^{i}\right]=i \epsilon^{i j} J_{l+m}^{j}+\frac{2 m}{P} J_{l+m}^{i}, \quad i, j=1,2 .
$$

The appearance of the second term in eq.(38) shows the distortion of the $\hat{E}_{2}^{c}$ chiral algebra since it would require only the first term. This modification of the chiral algebra in the light cone gauge is not a particular property of the Nappi Witten string: it happens even in the case of strings on flat Minkowski space. Indeed, even in this case, the commutators between the Fourier coefficients of the chiral currents belonging to the transverse coordinates, $\partial x^{A}$, and the Fourier coefficients of the dependent light cone coordinate, $\partial V$, (as computed from the vanishing of the world sheet energy momentum tensor), are entirely analogous to the second term in eq.(38).

We can rephrase the change of the chiral algebra in the light cone gauge in a slightly different way by introducing the quantities

$$
W_{n}=\frac{1}{4 k}\left(:\left(J^{1}\right)^{2}:_{n}+:\left(J^{2}\right)^{2}:_{n}\right)-\frac{P^{2}}{4} \delta_{n, 0}
$$

The commutator

$$
\left[W_{m}, W_{n}\right]=(m-n) W_{m+n}+\frac{2}{12} m\left(m^{2}-1\right) \delta_{m+n}
$$

shows that one can think of $W_{n}$ as a sort of light cone Virasoro generator with central charge 2. However the analog of eq.(38),

$$
\left[W_{m}, J_{n}^{i}\right]=-n J_{n+m}^{i}-\frac{i}{2} P \epsilon^{i j} J_{n+m}^{j}, \quad i, j=1,2
$$

means that the currents, $J_{n}^{i}$, are not purely weight one primary fields with respect to this Virasoro algebra.

In the same time we emphasize, that the 'zero mode algebra' generated by $J_{0}^{k}$, stays undistorted. Furthermore, since $J_{0}^{4}=-Q^{v_{0}}$ and $J_{0}^{i}=Q^{\delta_{i}}$ for $i=1,2$, this zero mode algebra is identical to the global, chiral $E_{2}^{c}$ one generated by $-Q^{v_{0}}, Q^{\delta_{i}}$ and $Q^{u_{0}}-Q^{\alpha} / 2$. This shows, that the zero mode operators of the string, $\Lambda$ and $\Lambda^{\dagger}$, play a multiple role: in addition to describing the CM motion of the string they also determine the step operators in these global, chiral $E_{2}^{c}$ algebras. Repeating the light-cone analysis with the antiholomorphic $\hat{E}_{2}^{c}$ current one finds in precisely the same way that the undistorted algebra of $\bar{J}_{0}^{k}$ is nothing but the one generated by $-Q^{v_{0}}, Q^{\Delta_{i}}$ and $Q^{u_{0}}+Q^{\alpha} / 2$. 
These findings have some interesting implications if we want to make a comparison between the physical states obtained here and in the covariant formulation. First, one has to identify $t$, the eigenvalue of the central element, $T_{0}$, on physical states with $k P$, the eigenvalue of $-Q^{v_{0}}$, and $j_{L, R}$, the eigenvalue of $J_{0}^{L, R}$, with the eigenvalue of $-\left(Q^{u_{0}} \pm \frac{1}{2} Q^{\alpha}\right)=-\frac{1}{P}\left(H \mp \frac{P}{2} Q^{\alpha}\right)$. Recalling that in the light-cone formalism the left-right symmetry condition for physical states can be written in the form of eq.(33), and combining this with the Hamiltonian, eq.(26), one readily shows that the eigenvalues of $-\frac{1}{P}\left(H \mp \frac{P}{2} Q^{\alpha}\right)$ on physical states depend on the excitation numbers of oscillators belonging to only one of the light-cone directions. Furthermore, taking the upper sign say and denoting the excitation numbers of the $k_{n}^{+}, \lambda_{n}^{+}$and $\alpha_{n}^{+}$oscillators by $M_{n}^{+}, L_{n}^{+}$and $C_{n}^{+}$ respectively, as well as introducing $S_{n}^{+}=M_{n}^{+}+L_{n}^{+}+C_{n}^{+}$, one finds for the eigenvalue:

$$
j_{+}=\frac{2 \sum_{n} S_{n}^{+}}{P}+\sum\left(M_{n}^{+}-L_{n}^{+}\right)+n_{\Lambda}+\frac{p_{A}^{2}}{4 P}-\frac{1}{2} k b P+\frac{a}{P} .
$$

Here $a$ is the normal ordering constant coming from the Hamiltonian, and all the oscillator excitation numbers, $M_{n}^{+}, L_{n}^{+}, C_{n}^{+}$and $n_{\Lambda}$ are non negative integers. This $j_{+}$describes a highest weight vector of the aforementioned $E_{2}^{c}$ algebra, if $n_{\Lambda}=0$. The highest weight values of $j_{+}$can be compared to the ones obtained in the covariant formalism [9], with the conclusion, that for $d=22$ (i.e. in the critical dimension) the two sets of numbers coincide.

\section{$\underline{6 . \text { Going beyond the } 0<P / 2<1 \text { restriction }}$}

Using the covariant quantization we proved, that in 26 dimensions the Nappi Witten string theory is free of negative norm states iff the continuous parameter, that characterizes the eigenvalue of the central element of the $E_{2}^{c}$ algebra on the physical states, falls into a definite, finite range. The analog of this parameter in the light cone quantization is the parameter $P$. Therefore it is interesting to investigate if some sort of restriction on $P$ arises even in the light cone gauge.

Repeating the procedure leading to eq.(21-24) when $N_{0}<P / 2<N_{0}+1$ with $N_{0}$ being a positive integer we found:

$$
\begin{aligned}
x^{1}+i x^{2} & =\frac{1}{\sqrt{k}}\left[e^{i \frac{P}{2} \tau}\left(\sum_{N_{0}+1}^{\infty} \frac{\lambda_{n}^{+}}{n-P / 2} e^{-i n(\tau+\sigma)}+\sum_{-N_{0}}^{\infty} \frac{\left(k_{n}^{+}\right)^{\dagger}}{n+P / 2} e^{i n(\tau+\sigma)}\right)\right. \\
& \left.+e^{-i \frac{P}{2} \tau}\left(\sum_{-N_{0}}^{\infty} \frac{\lambda_{n}^{-}}{n+P / 2} e^{-i n(\tau-\sigma)}+\sum_{N_{0}+1}^{\infty} \frac{\left(k_{n}^{-}\right)^{\dagger}}{n-P / 2} e^{i n(\tau-\sigma)}\right)\right]
\end{aligned}
$$


and

$$
\begin{aligned}
x^{1}-i x^{2} & =\frac{1}{\sqrt{k}}\left[e^{-i \frac{P}{2} \tau}\left(\sum_{N_{0}+1}^{\infty} \frac{\left(\lambda_{n}^{+}\right)^{\dagger}}{n-P / 2} e^{i n(\tau+\sigma)}+\sum_{-N_{0}}^{\infty} \frac{k_{n}^{+}}{n+P / 2} e^{-i n(\tau+\sigma)}\right)\right. \\
& \left.+e^{i \frac{P}{2} \tau}\left(\sum_{-N_{0}}^{\infty} \frac{\left(\lambda_{n}^{-}\right)^{\dagger}}{n+P / 2} e^{i n(\tau-\sigma)}+\sum_{N_{0}+1}^{\infty} \frac{k_{n}^{-}}{n-P / 2} e^{-i n(\tau-\sigma)}\right)\right]
\end{aligned}
$$

where the Fourier coefficients satisfy an equation, entirely analogous to eq.(2324):

$$
\begin{array}{lll}
{\left[k_{n}^{+},\left(k_{m}^{+}\right)^{\dagger}\right]=\delta_{n, m}(n+P / 2),} & {\left[\lambda_{n}^{-},\left(\lambda_{m}^{-}\right)^{\dagger}\right]=\delta_{n, m}(n+P / 2),} & n, m \geq-N_{0}, \\
{\left[k_{n}^{-},\left(k_{m}^{-}\right)^{\dagger}\right]=\delta_{n, m}(n-P / 2),} & {\left[\lambda_{n}^{+},\left(\lambda_{m}^{+}\right)^{\dagger}\right]=\delta_{n, m}(n-P / 2),} & n, m \geq N_{0}+1 .
\end{array}
$$

Taking at face value eq.(41-43) reveal only that in both light cone directions the number of $n+P / 2$ modded oscillators "exceeds" the number of $n-P / 2$ modded ones but show no problem with any sort of normalization for any value of $N_{0}$. However if one keeps track of the Fourier coefficients of $e^{ \pm i \frac{P}{2} \tau} e^{ \pm i n(\tau \pm \sigma)}$ for $0<n<N_{0}$ as $P$ changes from $0<P / 2<1$ to $N_{0}<P / 2<N_{0}+1$ then one learns, that the excess of $n+P / 2$ modded oscillators over the $n-P / 2$ modded ones is obtained by changing the interpretation of creation and annihilation operators for those $n-P / 2$ modded oscillators, that, formally, on the basis of eq.(23), would be "wrongly" quantized. As an example consider the $\lambda_{1}^{+},\left(\lambda_{1}^{+}\right)^{\dagger}$ pair, the coefficients of $e^{i \frac{P}{2} \tau} e^{ \pm i(\tau+\sigma)}$ in eq.(21-22), when $P / 2>1$. On the basis of eq.(23) there would be a negative number on the right hand side of their commutator, however writing $1-P / 2$ as $-(-1+P / 2)$ and interpreting $-\lambda_{1}^{+}$ as $\left(k_{-1}^{+}\right)^{\dagger}$ and $-\left(\lambda_{1}^{+}\right)^{\dagger}$ as $k_{-1}^{+}$we get precisely the form required by eq.(41-43). The necessity of changing creation and annihilation operators into each other (that corresponds to changing the ground state) when $P / 2$ is increased by one unit is the light cone equivalent of the presence of negative norm states in the covariant quantization when the particular parameter is outside of the allowed domain.

\section{$\underline{\text { 7. One loop partition function, modular properties }}$}

A bonus of the light-cone quantization in string theory is that it makes the computation of the one loop partition function easier, since in this gauge only the positive norm, physical, transverse states contribute. If we think of the one loop world sheet, the torus, characterized by a complex parameter, $\zeta$, as a cylinder of length $2 \pi \operatorname{Im} \zeta$ whose ends are identified, then one can twist the two ends relative to each other by an angle $2 \pi \operatorname{Re} \zeta$ before joining them. The operator which generates this twist is the one that generates the 'rigid' translation of the $\sigma$ coordinate, i.e. the one that appears on the left hand side of eq.(8), eq.(30), and 
eq.(33). Denoting this operator as $\Pi$, the complete one loop partition function is given by:

$$
\Theta(\zeta, \bar{\zeta})=\operatorname{Tr} e^{2 \pi i \operatorname{Re} \zeta \Pi} e^{-2 \pi \operatorname{Im} \zeta H}=\operatorname{Tr}\left(e^{\pi i \zeta(\Pi+H)} e^{-\pi i \bar{\zeta}(H-\Pi)}\right) .
$$

Using the explicit form of the Hamilton operator, eq.(26), and the form of $\Pi$ given by eq.(33), we see, as a novel feature, that both $H+\Pi$ and $H-\Pi$ contain both the left and the right moving oscillators. Indeed as

$$
H=L_{0}+\bar{L}_{0}+\hat{a}, \quad \Pi=L_{0}-\bar{L}_{0}-\frac{P}{2} Q_{\alpha},
$$

where the chiral $L_{0}\left(\bar{L}_{0}\right)$ operators are the zero index members of the two "lightcone" Virasoro algebras built from $W_{n}$ (respectively $\bar{W}_{n}$ ) and from the chiral parts of the $x^{A}$ oscillators:

$$
L_{n}=W_{n}+\frac{1}{2} \sum_{m}: \tilde{\alpha}_{n-m}^{-A} \tilde{\alpha}_{m}^{-A}:
$$

one can see that here - in contrast to the usual situation - $\Pi$ is not just the difference between the left and right null Virasoro generators. As a consequence both $H-\Pi$ and $H+\Pi$ contain the operator $\frac{P}{2} Q_{\alpha}$ that depends on both type of light cone oscillators. Therefore, if we denote the trace over one set of zero mode, one set of $n+P / 2$ modded and one set of $n-P / 2$ modded oscillators in the combination given by eq.(44) as

$$
\chi(q, \bar{q})=\frac{1}{\left(1-(q \bar{q})^{P / 4}\right)} \prod_{n=1}^{\infty}\left(1-q^{n}(q \bar{q})^{P / 4}\right)^{-1}\left(1-q^{n}(q \bar{q})^{-P / 4}\right)^{-1},
$$

$\left(q=e^{2 \pi i \zeta}\right)$, then, for fixed $P$, the one loop partition function takes the form:

$$
\Theta(\zeta, \bar{\zeta})=\frac{\eta(q)^{-d} \bar{\eta}(q)^{-d}}{(\operatorname{Im} \zeta)^{d / 2}}(q \bar{q})^{\frac{1}{2}\left(-b P^{2}-\frac{2}{12}+1+\frac{P}{2}\left(1-\frac{P}{2}\right)\right)} \chi(q, \bar{q}) \bar{\chi}(q, \bar{q})
$$

(In eq.(46) $\eta(q)$ denotes the Dedekind function). We emphasize that in deriving eq.(46) we encountered no divergent sums and correspondingly no regularization problem unlike in the analogue computation in the covariant quantization [5]. We obtain this nice feature because the non vanishing $P$ acts as a regulator in eq.(45), however in the $P \rightarrow 0$ limit $\chi(q, \bar{q})$ becomes singular: $\chi(q, \bar{q}) \mapsto$ $1 /(P \pi \operatorname{Im} \zeta) \prod\left(1-q^{n}\right)^{-2}$.

It is easy to see that $\Theta(\zeta, \bar{\zeta})$ is invariant under the $\zeta \rightarrow \zeta+1$ transformation. However the other generating transformation of the modular group, $\zeta \rightarrow-\frac{1}{\zeta}$, does not leave $\Theta$ invariant. Intuitively it is easy to understand the 
reason behind this : this transformation exchanges the $\tau$ and $\sigma$ directions and the fields described by eq. $(21,22)$ exhibit different boundary conditions in these directions: they are periodic in $\sigma$ but in $\tau$, because of the $e^{ \pm i \frac{P}{2} \tau}$ factors, they are only quasiperiodic.

Since modular invariance is a crucial requirement for a consistent string theory, we also derived the contribution of the $x^{i}$ coordinates to the one loop partition function using the Euclidean functional integral approach, where the boundary conditions appear in an alternative form.

After continuing the time coordinate $\tau \rightarrow-i T$ in the light cone form of the action, eq.(9), and introducing the

$$
z=\sigma+i T, \quad \bar{z}=\sigma-i T, \quad \zeta=\tau_{1}+i \tau_{2}
$$

parametrization of the world sheet torus, we used the complete system of functions:

$$
\psi_{(n, m)}(z, \bar{z})=\exp \left(\frac{\pi}{\tau_{2}}(n(z-\bar{z})+m(\zeta \bar{z}-\bar{\zeta} z))\right)
$$

periodic in both directions: $\psi_{(n, m)}(z+1, \bar{z}+1)=\psi_{(n, m)}(z, \bar{z}) ; \psi_{(n, m)}(z+\zeta, \bar{z}+$ $\bar{\zeta})=\psi_{(n, m)}(z, \bar{z})$ and the standard procedure of $\zeta$ function regularization (see e.g. [10]), to evaluate the determinant $\operatorname{det}\left(\mathcal{D}^{(+)} \mathcal{D}^{(-)}\right)$, where

$$
\mathcal{D}^{( \pm)}=-4 \partial_{z} \partial_{\bar{z}}+P^{2} \pi^{2} \pm 2 \pi i P\left(\partial_{z}+\partial_{\bar{z}}\right)
$$

This way we got

$$
\left(\operatorname{det}\left(\mathcal{D}^{(+)} \mathcal{D}^{(-)}\right)\right)^{-1 / 2}=(q \bar{q})^{\frac{P}{4}-\frac{1}{12}} \chi(q, \bar{q}) \bar{\chi}(q, \bar{q})
$$

for the $x^{i}$-s partition function. Since eq.(47) was obtained by using manifestly periodic boundary conditions, and it reproduces (apart from a not very interesting factor) the contribution of the $x^{i}$ coordinates to eq.(45-46), we conclude, that the modular non invariance of $\Theta(\zeta, \bar{\zeta})$ in eq.(46) is not the result of choosing improper boundary conditions.

Possibly, to obtain a modular invariant partition function, - especially the one suggested in ref.[5], - one should introduce some sort of "winding (and/or twisted) sectors" conceivably together with some appropriate projections. For these new sectors we need new ground states in the operator formalism, or appropriate "instanton" solutions in the functional integral formalism. A detailed investigation of this problem is beyond the scope of the present paper and we merely note that a candidate for the new ground state is provided by

$$
\left(x^{1} \pm i x^{2}\right)(\sigma)=\exp \left(\mp \frac{i P}{2}\left(\sigma-\sigma_{0}\right)\right)
$$


giving a static solution of eq.(6). It is straightforward to show that this solution, (just like the $x^{i} \equiv 0$ ground state used so far), - when augumented by $x^{A} \equiv 0$ - saturates the lower bound, $\left(-k b P^{2} / 2\right)$, of the Hamiltonian, eq.(11). As it stands, this solution is not periodic on $(0,2 \pi)$, nevertheless it's form guarantees, that no surface term arises in the case of periodic variations. It is also worth pointing out that this solution is not invariant under separate $\sigma$ translations and $x^{i}$ rotations, however an appropriate combination of these global symmetry transformations does leave it invariant.

\section{Conclusions}

In this paper we reviewed the main points of the quantization of the Nappi Witten string in the light cone gauge. It was shown, that imposing this gauge in an appropriately transformed form of the Nappi Witten background linearizes the equations of motion for the relevant degrees of freedom. Analyzing and quantizing these equations we exhibited the presence of two sets of twisted oscillators in both light-cone directions, deduced the energy and mass spectra and uncovered the global symmetries. Among the novel features it was found that the string's CM coordinate executes an oscillatory motion in the two transverse directions that correspond to the spatial part of the Nappi Witten metric. In addition it was also pointed out that the operators corresponding to these CM oscillations also act as the step operators of the global $E_{2}^{c} \times E_{2}^{c}$ symmetry algebra. It was demonstrated and explained why the critical dimension cannot be obtained from the usual argument in the operator formalism used in the present paper. We also showed, that the limitations on the range of the $t$ parameter, found in the covariant framework from the absence of negative norm states, follow also in the light cone formalism if we insist on the existence of a single ground state.

\section{Acknowledgements}

Three of us (P.F, Z.H and L.P) would like to thank Tours University for the hospitality and the Hungarian National Science and Research Foundation (Grant No. 2177) for a partial financial support.

\section{References}

[1] D. Amati and C. Klimcik, Phys. Lett. B219, 443 (1989); H. de Vega and N. Sanchez, Nucl. Phys. B317, 706 (1989); A.A. Tseytlin, Phys. Lett. B288, 279 (1992); Nucl. Phys. B390, 153 (1993); Phys. Rev. D47, 3421 (1993); C. Duval, Z. Horváth and P.A. Horváthy, Phys. Lett. B313, 10 (1993); E.A. Bergshoeff, R. Kallosh, T. Ortin, Phys. Rev. D47, 5444 (1993); R.E. Kallosh, A.D. Linde, T.M. Ortin, A.W. Peet, A. van Proyen, Phys. Rev. D46, 5278 (1992); K. Sfetsos, Phys. Lett. B324, 335 (1994); K. Sfetsos and A. Tseytlin, Nucl. Phys. B427, 245 (1994); C. Klimcik and A. Tseytlin, Phys. Lett. B323, 305 (1994). 
[2] G.T. Horowitz and A.R. Steif, Phys. Rev. D42, 1950 (1990); A. Steif, Phys. Rev. D42, 2150 (1990).

[3] C. Nappi and E. Witten, Phys. Rev. Lett. 71, 3751 (1993).

[4] E. Kiritsis and C. Kounnas, Phys. Lett. B320, 264 (1994).

[5] E. Kiritsis, C. Kounnas and D. Lüst, Phys. Lett. B331, 321 (1994).

[6] P. Goddard and C.B. Thorn, Phys. Lett. B40, 235 (1972).

[7] R. Hwang Nucl. Phys. B354, 100 (1991); J. Balog, L. O'Raifeartaigh, P. Forgács and A. Wipf, Nucl. Phys. B325, 225 (1989).

[8] C.B. Thorn, Nucl. Phys. B286, 61 (1987).

[9] P.Forgács, Z. Horváth and L. Palla, unpublished

[10] P. Ginsparg, In "Fields, strings and Critical Phenomena" ed. by E. Brézin, and J. Zinn-Justin, Elsevier Science Publishers, B. V. (1989).

[11] R.E. Rudd, Nucl. Phys. B427, 81 (1994); 